\title{
Management of Bilateral Spontaneous Pneumothorax Related to COVID-19 Infection
}

\author{
Rachel Gardner BA, Aaron Tipton MD, Matthew Janko MD, Mohammad Usman MD, Philip Linden MD, Christopher Towe MD, \\ Stephanie Worrell MD, Luis Argote Greene MD*
}

Department of Thoracic Surgery, University Hospitals Cleveland Medical Center, Cleveland, USA.

*Corresponding author: Luis Argote-Greene, Department of Thoracic Surgery, University Hospitals Cleveland Medical Center, Cleveland, USA.

Received Date: July 16, 2021: Accepted Date: July 26, 2021: Published Date: July 29, 2021

Citation: R Gardner, A Tipton, M Janko, P Linden, Luis A Greene, et al. (2021) Management of Bilateral Spontaneous Pneumothorax Related to COVID-19 Infection. Journal of Clinical Surgery and Research. 2(3) DOI: 10.31579/2768-2757/022

Copyright: @2021 Luis Argote-Greene, This is an open-access article distributed under the terms of the Creative Commons Attribution License, which permits unrestricted use, distribution, and reproduction in any medium, provided the original author and source are credited.

\begin{abstract}
Spontaneous pneumothorax is a rare but potentially fatal complication of coronavirus infection and COVID-19 pneumonia. Data is limited to guide clinicians in the setting of spontaneous pneumothorax when conservative management fails and operative intervention is required. We report the clinical course and operative strategy for a patient who presented with sequential bilateral spontaneous pneumothorax after being diagnosed with severe COVID-19 pneumonia. Conservative management with bilateral tube thoracostomies failed to resolve persistent alveolar-pleural fistulae, and ultimately the patient recovered after synchronous bilateral VATS blebectomies in the operating room. The timing and treatment of spontaneous pneumothorax requires follow-up education and surveillance for patients with this disease, as lack of prompt treatment outside the hospital could increase patient mortality. Spontaneous pneumothorax is a concern in patients who re-present with recurrent symptoms after COVID-19 diagnosis. When conservative treatment fails, surgery can safely be performed in COVID-19 patients with simultaneous bilateral pneumothorax with full functional recovery.
\end{abstract}

Keywords: covid pneumothorax; spontaneous pneumothorax; bilateral pneumothorax; covid pneumonia

\section{Introduction}

Severe acute respiratory syndrome coronavirus 2 (SARS-CoV-2) causes a systemic infection (COVID-19) that is associated with barotrauma in $15 \%$ of ventilated patients including both pneumothorax (PTX) and pneumomediastinum [1]. Limited data is available for management of COVID-19 patients with spontaneous pneumothorax (SPTX) [2]. Medical management alone may be related to prolonged hospitalization, intubation, and additional complications [3, 4]. Here, we present a COVID-19 patient with bilateral SPTX treated successfully with thoracoscopy and bleb resection.

\section{Case Presentation}

A 62-year-old male non-smoker with chronic untreated hepatitis $\mathrm{C}$ was diagnosed with COVID-19 by nasal swab in May 2020. He presented with fever, tachycardia, and hypoxia requiring supplemental oxygen by nasal cannula. He was treated with Remdesivir 200 milligrams (mg) intravenously (IV) followed by $100 \mathrm{mg}$ IV daily for five days. No PTX was seen on computed tomography (CT). He was discharged after seven days.

He re-presented 17 days later with shortness of breath, chest pain, tachypnea, hypoxia, and mild leukocytosis. Repeat COVID-19 nasal swab was negative. An echocardiogram demonstrated right heart strain. CT demonstrated a right tension PTX without evidence of pulmonary embolism (Figure 1). A right pleural thoracostomy tube facilitated reexpansion of the right lung. Intravenous cefepime was initiated for possible hospital-acquired pneumonia. Nasal cannula oxygen was supplemented. On hospital day two, a new left pneumothorax was discovered and a left pleural thoracostomy tube was placed, resolving the pneumothorax. Water-seal trials showed persistent alveolar-pleural leaks, likely secondary to ruptured blebs from cystic lung changes. 


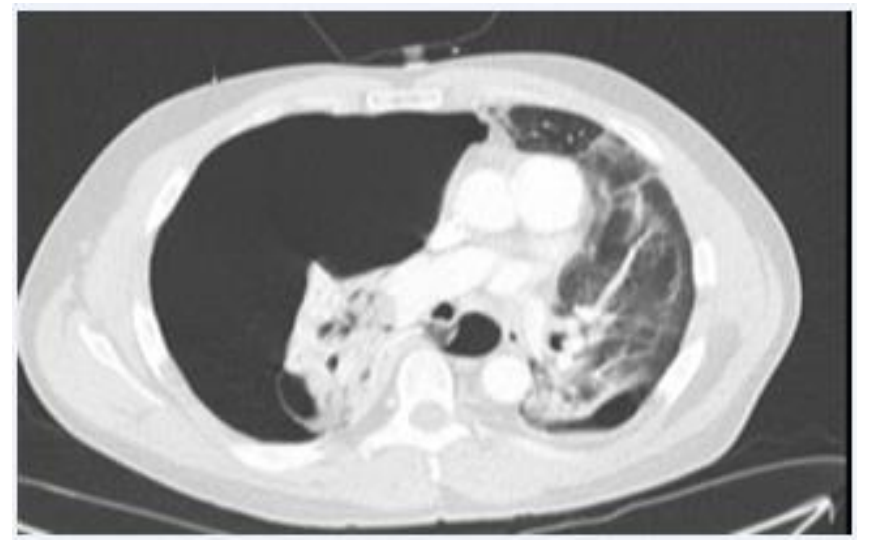

Figure 1: Spontaneous Pneumothorax Two Weeks after COVID-19 Diagnosis

After 12 days of failing to progress with persistent air leaks and pleural effusions, the patient underwent video-assisted thoracoscopic surgery (VATS), left lower and upper lobe blebectomies, and a right upper lobe wedge resection. The previously-placed small diameter right thoracostomy tube was exchanged and connected to suction with the patient on double-lung ventilation in the supine position. The left lung was then isolated in the right lateral decubitus position, and a left $6^{\text {th }}$ intercostal space mini-thoracotomy was performed. Copious serosanguineous pleural fluid was drained. A thickened visceral pleura contained such a sizable sub-pleural accumulation of sanguineous fluid that the parenchyma floated inside the sac. The visceral pleural sac and affected lung were removed with Endo-GIA purple load staplers
(Covidien, Dublin, Ireland). Similar, but less dramatic, findings were seen on the contralateral lung. Bilateral chest-tubes remained in place after the operation.

The postoperative course was uncomplicated. No further alveolar-pleural leaks were detected. Chest tubes were removed three days later. The patient was discharged home on postoperative day seven without supplemental oxygen. Histological examination of the operative specimens demonstrated pulmonary blebs with organizing pleuritis, subpleural hemorrhages, and fibrosis. Two months later, he had recovered well with improved exercise tolerance. Follow-up CT demonstrated postsurgical changes and non-specific interstitial opacities (Figure 2).

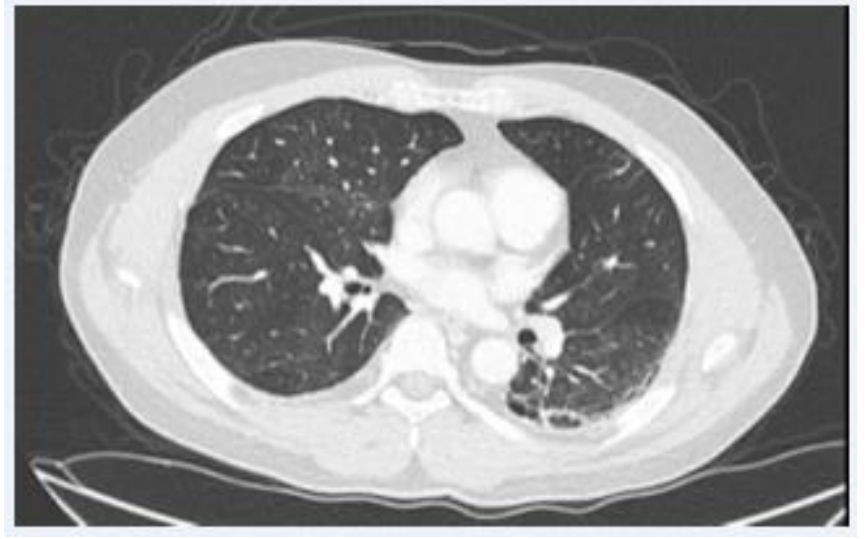

Figure 2: Complete Resolution of Bilateral Pneumothorax Postoperatively

\section{Discussion and Conclusions}

Approximately $1 \%$ of patients with COVID-19 develop SPTX [5]. Bellini reported the first two cases of COVID-19 patients with SPTXs treated surgically [3]. Like our patient, both patients were readmitted weeks after initial hospitalization. The authors concluded that blebectomy and wedge resection were safe and efficacious alternatives to conservative management. Aiolfi also reported two COVID-19 patients with unilateral SPTXs who were successfully treated with thoracoscopy and bleb resection [4]. While ideal timing of thoracoscopy in COVID-19 patients with persistent pneumothorax remains unclear, operative intervention under one week from chest tube drainage led to air leak control, contributing to a faster recovery.

The thickened, stiff parenchyma found in COVID-19 lungs prevents proper function, requires prolonged mechanical ventilation, and predisposes to complications such as interstitial pneumonia, acute respiratory distress syndrome, and PTX $[1,4]$. The authors suggest that
COVID-19 patients be informed of their risk for subsequent complications, such as SPTX, after resolution of their acute symptoms, and seek medical attention if they experience chest pain or shortness of breath in the weeks following their diagnosis.

SPTX may occur weeks after a COVID-19 diagnosis. When conservative treatment fails, surgery may safely be performed in COVID-19 patients with simultaneous bilateral SPTX.

Conflicts of Interest: No conflicts of interest.

\section{Funding Sources: None}

\section{References}

1. Martinelli AW et al. (2020) COVID-19 and pneumothorax. Eur Respir J. 56(5). 
2. Wang W et al. (2020) COVID-19 with spontaneous pneumothorax, pneumomediastinum and subcutaneous emphysema. J Travel Med. 7(5).

3. Bellini R et al. (2020) Spontaneous pneumothorax as unusual presenting symptom of COVID-19 pneumonia. J Cardiothorac Surg. 15(1).
4. Aiolfi A et al. (2020) Management of Persistent Pneumothorax With Thoracoscopy and Bleb Resection in COVID-19 Patients. Ann Thorac Surg. 110(5).

5. Chen $\mathrm{N}$ et al. (2020) Epidemiological and clinical characteristics of 99 cases of 2019 novel coronavirus pneumonia in Wuhan, China. Lancet. 395(10223).
This work is licensed under Creative Commons Attribution 4.0 License

To Submit Your Article Click Here: Submit Manuscript

DOI: $10.31579 / 2768-2757 / 022$
Ready to submit your research? Choose Auctores and benefit from:

* fast, convenient online submission

* rigorous peer review by experienced research in your field

* rapid publication on acceptance

* authors retain copyrights

* unique DOI for all articles

* immediate, unrestricted online access

At Auctores, research is always in progress.

Learn more https://auctoresonline.org/journals/journal-of-surgical-casereports-and-images 